\title{
An Integrated Approach for Knowledge discovery and Information retrieval on Web
}

\author{
${ }^{1}$ MOHAMMAD SHAHID, ${ }^{2}$ Dr. ANIL KUMAR SRIVASTAVA \\ ${ }^{1}$ Research scholar, Dept. of Computer Science \& Engineering OPJS University Churu Rajasthan, \\ India, ${ }^{1}$ mohd86shahid@gmail.com, ${ }^{2}$ anil.kumar@gmail.com
}

\begin{abstract}
This Paper focuses on the integration of web information and subsequent knowledge relationship discovery within the integrated web data. The problem of information overload on the Internet has brought new attention to the ideas of filtering information on internet. Knowledge Discovery is often used for analysis of large amounts of web data and enables addressing a number of tasks that arise in Semantic Web and require scalable solutions. The World Wide Web and related web Information resources no arguably stand as the best-preferred medium for distributing information. It introduces various approaches to knowledge relation discovery like model creation, exact comparison and dynamic comparison. The nature of the web and the mass of valuable web information it holds, poses an ideal stage for applying data mining techniques for efficient discovery of knowledge from the World Wide Web. The eagerness shown by various research communities has made web based data mining (Web Mining) a rich mixture of different technologies. Therefore the heterogeneity in the area of web mining is as high as web itself. Our objective is to design an approach for information filtering, a general approach to personalized information filtering. Social Information filtering essentially automates the process of "word-of-mouth" recommendations: items are recommended to a user based upon values assigned by other people with similar taste. The system determines which users have similar taste via standard formulas for computing statistical correlations. The World Wide Web (WWW) provides a vast source of information. Technique for making personalized recommendations from any type of database to a user based on similarities between the interest profile of that user and those of other users. Recent years have seen the explosive growth of the sheer volume of information.
\end{abstract}

Keywords - Components of an Information Management Computer Network, Web usage mining, web Data, Web usage mining architecture.

\section{INTRODUCTION}

The size of the data is rapidly increasing day by day very quickly so we have managed and integrate. Now a day because of technologies its very easy to publish papers, journal and conferences proceedings and distributing information to the end users. All of us have known feelings of being overwhelmed by the number of new books, articles, journals, publications, conference proceedings coming out easy year. Because of technology advancement its very easy to spread information for users and to users with help of technology we can filter most valuable and relevant information to us. Because of overloaded information on internet has brought new idea of filtering information on internet has brought new attention of filtering information on internet. Every need to update his or her personal information is useful for his on her organization and self also needs some methods of making using internet. Filtering information using keys and looking for patterns or keywords in data and lettering user feedback guide future
So key required to filter the information on internet is sure: keywords in to data or looking for pattern matching Belkin and Croft (Belkin and Croft 1992) point out that at an abstract level, that Information retrieval for web and Information filter from are very similar. They elaborate that unstructured data and not from controlled database. The information is primary textual information and it is in large amount and is gradually incoming. They believes in filtering is based on users profile traditionally, typically show users long term interests and benefit . Filtering means removing unused data while searching information or get to identified only required information. So we need technology to help us in a better way of filtering data for useful information.

Text oriented data are web documents mostly. And almost all relevant information are embedded in the text and could not be explicitly or specified in user query We know that important information is always highlighted by keywords or meaningful structures since good visual effects are common practice for representing Web data. Generally 
common patterns exist in English, for example we can take the word after "DR." or "Mrs." should be a name. Knowledge discovery of Internet data also known as web mining, has been an area of recent cross-disciplinary research interest For the discovery of patterns that are actionable in electronic commerce environment, web use mining that is mining of server $\log$ files and related marketing information has proved to be the most optimum structure .

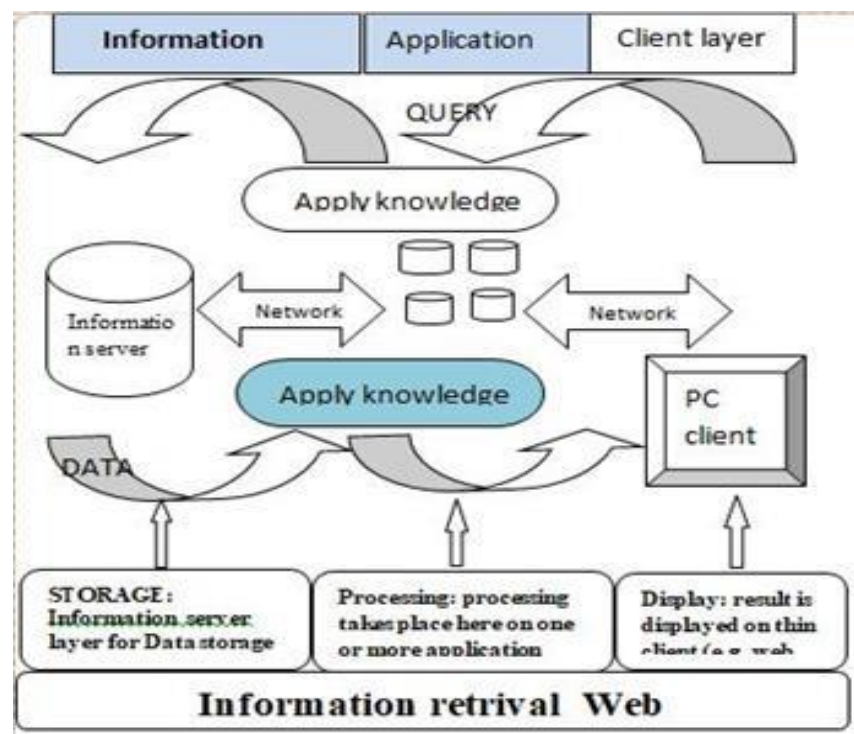

Figure 1.

\section{WEB DATA}

Knowledge Discovery in database is one of the key element to create a suitable target data set for data mining tasks. Data can be collected in web mining at the server side, client side ,proxy server obtained from or organised database(which can be having analytic business data or web analytic data termed web information )

Location of data source is not terms of collection different types of data, the data was collected from kinds of data variable, the segment of population and its methods implemented .In web mining many kinds of data can be used. So this paper classifies such data in the different following types.

Data Content: Web pages data was designed that is real data in web page to convey to the usaers, but not limited as consist of different text and graphics.

Structure Data: Basically the data which defines organisation of the context. Intra pages structured data which includes various types XML tags within provided pages, and various HTML defined tags.

Web DATA Log: Cookies logs, error logs and server logs are three types of log files. I which Server logs are either stored in the Common Log files Format or the more resent extended $\log$ files format.

1. Internet provider IP address: This can be either webminer.com or 204.58 .155 .58

2. Identification field: This usually appears as a dash, "-"

3. AuthUser: This is an ID or password for accessing a protected area

4. Date, time, and GMT (Greenwich Mean Time): Thu July 17 12:38:09 1999

\section{Transaction: Usually "GET" filename such as /index.html/products.htm}

6. Status or error code of transaction: Usually 200 (success)

7. Size in bytes of transaction (file size): 3234 Additional Fields in the Extended Log Format

8. Referrer: search engine and keyword used to find your Web site, such as http://search.yahoo.com/bin/search?p=data+miningý/index .html

9. Agent: browser used by your visitor, such as Mozilla/2.0 (Win95; I)

\section{Cookie: .snap.com TRUE / FALSE 946684799 u_vid_0_0 00ed7085}

Missing links will be stored in Error log so we can say that Error $\log s$ stored of failed request like authentication 
failures or timeout difficulties. Server data storage capacity difficulties or various link detection ---Which when satisfactory corrected so customer satisfaction can be seen surly. And the use of error logs for the discovery of actionable marketing intelligence has so proven. The client side held cookies are tokens generated by the web pages of web servers. The information stared on client side or client $\operatorname{logs}$ assist in order to ameliorate the transaction less state of web server interactions and enables web servers to track client access their hosted web pages. Cookies data is being customizable so that it goes hand to hand the structure and contents of marketing data.

The E-commerce that generates a fourth data source is query data to a web server. For example a online customer usually search a data from e-commerce webpages and the information of related items for web pages online any product or client to a research database may search for publications.

To access cookies data the logged query must linked with and or data registration in formation. For handling queries there is no formal drafts as standard although new specification suggestions have reached draft An Integrated Approach for Knowledge discovery and Information retrieval on Web stage for instance Resource Framework RFD. Generally we would make use of logical queries ,it would be grouped in to logical unit, for marketing related.

\section{WEB USAGE MINING}

From the web servers web usage mining is self-discovery of users access pattern. On the daily operation the organisation collects huge amount of information on daily basis operation, generates self by web servers and get collected in server access logs [7]. Other of user information counts referrer logs which contain information about the referring pages for each page reference and user registration on survey gathered data. Analysing such data can helps the organisation to study the value of each and every customer for life time. Other planning and marketing strategies across product and effectiveness of promotional campaigns and other things.it will provide information that how to restructure a web site to create more effectiveness of organisational presentation .For selling product and advertisement on internet, and more efficient techniques helps organization for targeting a new approach of business and per motion for business

\section{CONCLUSION}

The importance of the World Wide Web as an information source is by now a fact and needs to proof. However due to the underlying structure of the World Wide Web Information, retrieving meaningful information is an exhausting task. Hence from tradition query database the generated query on internet is enough different for example a RD relational databases, are static centralized and structured.
. In this paper we looked at how data mining (based on Machine Learning) has been implemented on the World Wide Web to extract Information (knowledge). Mainly in the field of "web content mining" we learnt much of the work could be categorized into three areas namely: Information Retrieval in unstructured data, Information Retrieval in semi-structured data and DB View of the World Wide Web. We looked at some selected work done in these three categories. Most of the traditional machine learning algorithms have been extended or combined with techniques from other fields such as Natural language processing or constraint programming. We also looked at two of the future trends of the World Wide Web where machine learning could be applied. The web presents a heterogeneous, distributed, huge and still growing database for the data-mining researcher. The opportunities for mining in this ocean of information (knowledge) are endless. We looked at many future directions which came up as a result of this survey.

\section{REFERENCES}

[1] Chia-Hui Chang and Chi Hsu - Enabling Concept based Relevance feed back for information retrieval on the WWW, 2009

[2] Kerlocker, J. Konstan, J. Nrochers, A. and Riedl, J. - An Algorithmic Framework for Performing Collaborative Filtering. In proceedings of ACM SIGIR'99. ACM press, 1999.

[3] Sarwar, B. M., Karypis, G., Konstan, J.A., Reidl, J. Analysis of Recommendation Algorithms for E-commerce. In Proceedings of the ACM EC'00 Conference. Minneapolis, 2000.

[4] Tao Guan and Kam Fai Wong - KPS - A Web Information Mining Algorithm, 1999.

[5] Ruslana Svidzinska - A World Wide Web Meta Search Engine, 2005.

[6] Paiano, R.; Pasanisi, S. A New Challenge for Information Mining. Broad Res. Artif. Intell. Neurosci. 2017,8 63-80.

[7] D. Dou, H. Wang, H. Liu, Semantic data mining: A survey of ontologybased approaches, in: Semantic Computing (ICSC), 2015 IEEE International Conference on, IEEE, 2015, pp. 244-251

[8] M. Schmachtenberg, C. Bizer, H. Paulheim, Adoption of the Linked Data Best Practices in Different Topical Domains, in: International Semantic Web Conference, 2014

[9] ] Q.K. Quboa, M. Saraee, A state-of-the-art survey on semantic web mining, Intell. Inf. Manage. 5 (2013) 10

[10] Keßler, C.; d'Aquin, M.; Dietze, S. Linked Data for Science and Education. Semant. Web J. 2013, 4, 1-2

[11] IBM. Knowledge Discovery and Data Mining. Available online: http://researcher.watson.ibm.com/researcher/ view_group.php?id=144 (accessed on 8 June 2018).

[12] http://ubiquity.acm.org [13]

[13] http://www.mariapinto.es/ciberabstracts/Articulos/Knowled ge\%20Discovery.htm

14. http://www.hindawi.com/journals/ijdsn/2015/718390/tab1/ 